\title{
Comparing the Results of Three Ergonomic Assessment Tools
}

\author{
Ahmadi $\mathbf{M}^{1}$, Etemadinezhad $\mathrm{S}^{1 *}$, Yazdani Charati J ${ }^{2}$, Akbarzadeh $\mathrm{L}^{1}$ \\ and Kaveh $\mathbf{R}^{1}$ \\ 1Department of ergonomics and occupational health, Mazandaran University of Medical \\ Sciences, Iran \\ ${ }^{2}$ Department of Biostatistics, Mazandaran University of Medical Sciences, Iran
}

\section{Research Article}

Volume 2 Issue 4

Received Date: August 07, 2018

Published Date: August 24, 2018

DOI: $10.23880 /$ eoij-16000175

*Corresponding author: Siavash Etemadinezhad, School of Health Sciences, Mazandaran University of Medical Sciences, Khazar-Abad road, Sari, Iran, Tel: +98113354-3231; Email: dr.setemadi@yahoo.com

\section{Abstract}

The outcome of an ergonomic assessment underlies and is followed by the decisions on where and when an intervention is required; therefore, selecting a suitable method indirectly affects the effectiveness of an ergonomics program. Currently, available ergonomics assessment tools vary over a wide range, some of which can be interchangeably used for similar purposes. This could make the practitioners confuse to choose the most appropriate tool. QEC, ManTRA and MFA are of those methods having to do with routine manual tasks which their distinctions have never been drawn. The objective of this study was to compare the results of these three ergonomics methods to determine their agreements with one another and correlations with workers' musculoskeletal discomforts. The tools were applied to 350 manual tasks by several ergonomics experts, as was the Cornel Musculoskeletal Questionnaire to the workers working on those tasks. The outputs of the three methods were compared with one another as well as with the workers' musculoskeletal discomforts rate. Of the methods, MFA evaluated the tasks as at risk in more moderate way than the other methods and had the most correlation with the workers' musculoskeletal discomforts. ManTRA was also found as the most lenient approach which tended to ignore some risky tasks of the work systems. In contrast, QEC was the strictest of the three and would classify a task as high/very high risk more than the other tools in this study.

Keywords: Ergonomic Risk Assessment; QEC; ManTRA; Muscle Fatigue Assessment; Cornel Questionnaire

\section{Introduction}

Preventing musculoskeletal disorders requires eliminating or, at least, reducing the workers exposure to work physical risk factors $[1,2]$. To determine the degree of exposure to musculoskeletal risk factors and prioritize the required ergonomic interventions, they should be systematically analyzed [3]. During past decades, many tools have been developed to evaluate musculoskeletal risk factors $[4,5]$. These methods are categorized, mainly, into two observation and instrumentation-based methods. The observation methods have been more developed among the practitioners because of being inexpensive, easier to use and less interrupted as well as lack of need to special equipment [6]. These are classified into whole body assessment tools, upper limb assessment tools and manual material handling, of which the most appropriate one must be chosen to assess a task [7]. An appropriate risk assessment technique is challenging to select and depends on the type of the work, because each 


\section{Ergonomics International Journal}

technique has been developed to be relevant with a specific type of task or a cluster of risk factors $[4,8]$. Thus the practitioners have to properly specify the task's properties and risk factors to make a more accurate choice of the tools [9]. However, they will still probably encounter a few tools which can be interchangeably used for the same purpose. A trade-off between the similarpurpose tools should be made, so that the practitioners could be able to map them onto the task's characteristics. Most of the previous studies compared various ergonomic assessment tools, some of which belonged to different particular categories, as per mentioned above $[4,5,10]$. There are, however, two studies in which only the manual material handling techniques were compared $[8,11]$.

The whole body assessment tools evaluate the whole body exposure to work-related musculoskeletal risk factors. OWAS, REBA, MFA, PLIBEL, QEC, EAWS, PERA, ManTRA and ISO 11226 are examples of this category. Some of them just concentrate primarily on the working posture; others, however, take additional physical risk factors into consideration as well [6]. Quick Exposure Checklist (QEC), Muscle Fatigue Assessment (MFA) and Manual Task Risk Assessment (ManTRA) are of those tools which consider the work factors including Posture, Force, Load, Duration, Repetition, and Vibration in the analysis [12]. Although, QEC method has been investigated in many studies and its output data has been proved to be reliable [13-16]; MFA and ManTRA are rarely used in researches. Therefore, the aim of this paper was to compare the results of QEC, MFA and ManTRA methods to determine their agreements with one another and correlations with workers' musculoskeletal discomforts.

\section{Method}

This study was carried out in nine plants including two steel industries, two fish-canning factories, wheat flour plant, oil factory, industrial-machinery manufacturing company, heavy electrical equipment factory, schools equipment company and tobacco factory, providing more than 1000 jobs, most of which included manual tasks. The three ergonomics methods and the Cornell questionnaire were completed for a pilot sampling of 15 tasks, of which 35 percent were at high risk level. Then, based on calculation of the statistical formula, 350 tasks were considered as the sample size. All the tasks in the factories were preliminary inspected by several ergonomics experts to select those that contain manual tasks. The inclusion criteria for the workers were having at least one year work experience in that task and not to have any chronic musculoskeletal disease. The workers were informed about the purpose of the study and the confidentiality of the data. They were also explained what the musculoskeletal discomforts are and how to fill the Cornel Musculoskeletal Discomforts questionnaire. For each body part just the frequency multiplied by the discomfort severity yielded the Cornell questionnaire overall score. Each task was observed precisely and recorded as photos and videos by the ergonomists in order to assess the musculoskeletal risks using ManTRA, MFA and QEC tools.

\section{Assessment Tools}

Quick Exposure Checklist: QEC is an ergonomic assessment tool to quickly measure the exposure of four body regions (back, shoulder/arm, hand/wrist and neck) to the musculoskeletal risk factors. It considers the risk factors and work conditions including working body posture and movements, weight handled manually, hand force exertion, task duration, work pace, vibration and visual demands. Each body part gets a risk score separately which defines its risk level. QEC divides the risk levels into four levels as low, moderate, high and very high risk. The total score is the sum of each body part's score divided by maximum possible score which is 176 for manual material handling tasks and 162 for the others [17-19].

Manual Task Risk Assessment: ManTRA is an ergonomic audit tool which evaluates the exposure to musculoskeletal risk factors. It allows the inspectors to assess the five task conditions including total time, repetition, exertion, posture and vibration for each of four body regions (lower limbs, back, neck/shoulder and arm/wrist/hand) using a five-point scale. There are five codes for each region as five task characteristics' risk score. Repetition risk score is combined with duration and task cycle, both of which is already assessed. Also a combined score of force and speed is considered as Exertion risk. For each body region the combined cumulative score is calculated as the total risk score which ranges between 5 and 25. This technique just determines whether a task is at risk or safe. The task needs to be modified if either the exertion score equals 5 , the sum of exertion and awkward posture is 8 or greater, or the total risk is 15 or greater $[20,21]$.

Muscle Fatigue Assessment: MFA is a functional job analysis technique that was developed by Rodgers and Williams in 1987 to indicate workers' discomforts [22]. This method is used as a suitable risk predictor tool in the occupations with no specific biomechanical issues. It audits the work factors such as posture, force exertion, grip and manual handling as well as effort duration and 


\section{Ergonomics International Journal}

frequency in workplaces for entire body parts including neck, shoulders, back, arms/elbows, wrists /hands /fingers, legs/knees and ankles/feet/toes [23]. In this method, once the severity of each task effort, for each body part, is determined by the worker using a ten-point scale, the duration and the frequency of each effort are measured. The score of each parameter (effort, continuous effort duration and frequency) is measured using a four-point scale. The "priority for change" scores put the task risk into four levels as low, moderate, high and very high [18].

\section{Cornel Musculoskeletal Discomfort Questionnaire}

The Cornell musculoskeletal discomfort questionnaire that was developed in 1999 by Dr. Alan Hedge and colleagues is a data collection tool for musculoskeletal disorders. In this questionnaire, a worker expresses the past seven-day frequency of pains using a five-point scale for 12 body organs that are graphically illustrated. In case of any pain, the worker is required to explain its discomfort and interference with work ability using three-point scales $[24,25]$. The final score for each body part is obtained from multiplying the frequency score by the discomfort score by the interference score [26].

\section{Results}

Comparison of the musculoskeletal risk levels resulted from QEC, ManTRA and MFA is provided in Figure 1. For each body region high and very high risks, taken together, are presented as percentage of all total tasks. As an average of all risk percentages, MFA identified $52 \%$ of the tasks as unsafe compared with QEC with $67 \%$ and ManTRA with 23\%.

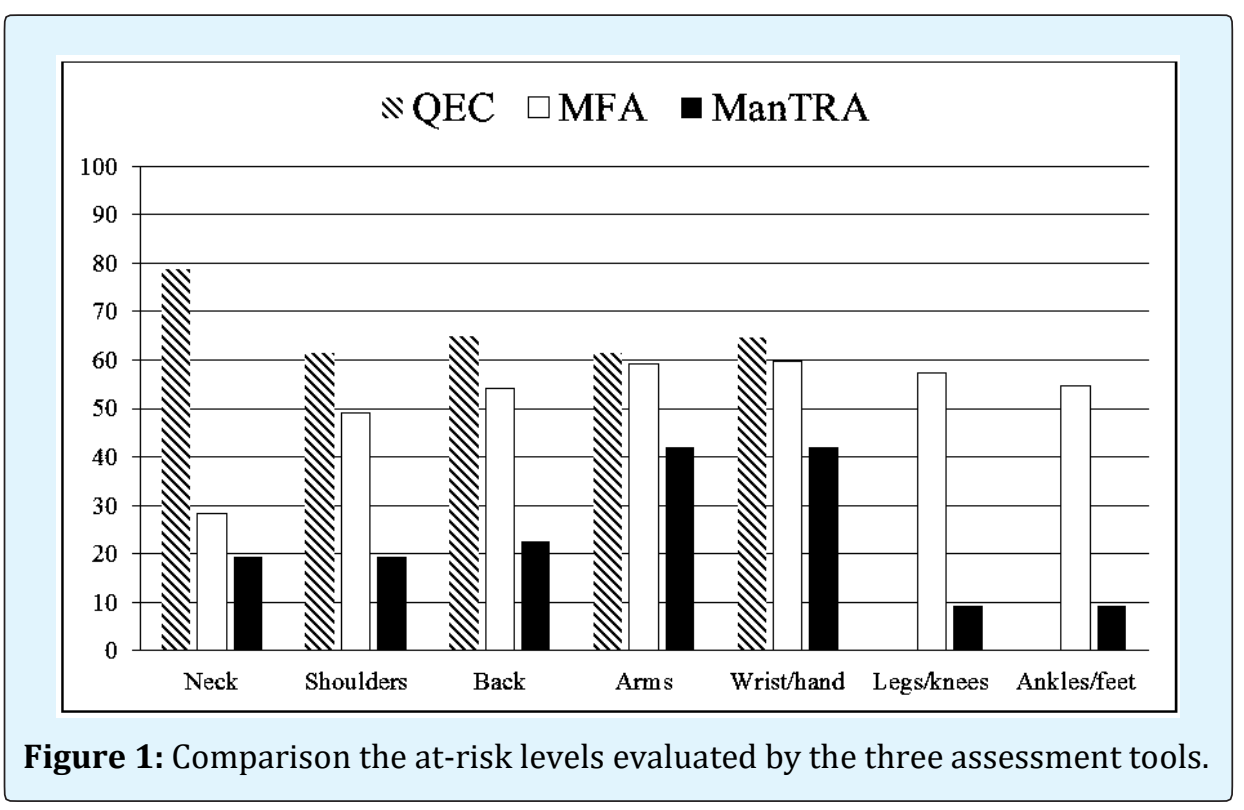

The degree of agreement and disagreement between the three methods was estimated by Kappa and Log-linear statistical tests at a significance level of 0.05 .

The Cohen's Kappa coefficient was calculated for each pair of methods with respect to each body part. Tables 1 to 3 demonstrate the results of Kappa test in which the value ranges from -1 to 1 and represents the extent to which agreement is reached between each pair of methods. The more negative the value is, the more negative correlation exists. The significant Positive value, on the contrary, shows positive correlation between the two methods. Also, there is no correlation when the value is 0 .

\begin{tabular}{|c|c|c|}
\hline Body region & Value & Approx. Sig \\
\hline Neck & -.594 & $<0.001$ \\
\hline Shoulders & -.420 & $<0.001$ \\
\hline Back & -.423 & $<0.001$ \\
\hline Arms & -.195 & $<0.001$ \\
\hline Wrist/hands & -.225 & $<0.001$ \\
\hline Total & -.404 & $<0.001$ \\
\hline
\end{tabular}

Table 1: Agreement between QEC and ManTRA using Kappa test. 


\section{Ergonomics International Journal}

\begin{tabular}{|c|c|c|}
\hline Body region & Value & Approx. Sig \\
\hline Neck & -.164 & $<0.001$ \\
\hline Shoulders & -.040 & .003 \\
\hline Back & -.033 & .009 \\
\hline Arms & -.007 & .555 \\
\hline Wrist/hands & -.015 & .233 \\
\hline Total & -.043 & $<0.001$ \\
\hline
\end{tabular}

Table 2: Agreement between QEC and MFA using Kappa test.

\begin{tabular}{|c|c|c|}
\hline Body region & Value & Approx. Sig \\
\hline Neck & -.025 & .012 \\
\hline Shoulders & -.090 & $<0.001$ \\
\hline Back & -.098 & $<0.001$ \\
\hline Arms & -.057 & $<0.001$ \\
\hline Wrist/hands & -.059 & $<0.001$ \\
\hline Leg/knees & -.144 & $<0.001$ \\
\hline Ankle/feet & -.135 & $<0.001$ \\
\hline Total & -.087 & $<0.001$ \\
\hline
\end{tabular}

Table 3: Agreement between ManTRA and MFA using Kappa test.

As shown in Table 4, the Log-linear analysis examined the methods seven times, each time with respect to a specific body region. It should be noted that MFA in this analysis is selected by SPSS software to serve as a basis for comparison with the results of the other tools. QEC also does not deal with the lower limbs. That is why; it was not considered in lower limbs-related analyses.

\begin{tabular}{|c|c|c|c|}
\hline \multirow{4}{*}{ Neck } & Method & Estimate & Significance \\
\hline \multirow{3}{*}{ Shoulders } & QEC & 1.02 & $<0.001$ \\
\cline { 2 - 4 } & ManTRA & -0.373 & 0.029 \\
\cline { 2 - 4 } & MFA & $0 \underline{\underline{a}}$ &. \\
\hline \multirow{3}{*}{ Back } & QEC & 0.222 & 0.046 \\
\cline { 2 - 4 } & ManTRA & -0.921 & $<0.001$ \\
\cline { 2 - 4 } & MFA & $0^{\underline{a}}$ &. \\
\hline \multirow{3}{*}{ Arms } & QEC & 0.178 & 0.098 \\
\cline { 2 - 4 } & ManTRA & -0.875 & $<0.001$ \\
\cline { 2 - 4 } & MFA & $0^{\underline{a}}$ &. \\
\hline \multirow{3}{*}{ Wrist/hands } & QEC & 0.04 & 0.71 \\
\cline { 2 - 4 } & ManTRA & -0.34 & 0.004 \\
\cline { 2 - 4 } & MFA & $0 \underline{\underline{a}}$ &. \\
\cline { 2 - 4 } & QECC & 0.077 & 0.464 \\
\cline { 2 - 4 } & MFTRA & -0.351 & 0.003 \\
\hline \multirow{2}{*}{ Leg/knees } & ManTRA & -1.813 & $<0.001$ \\
\cline { 2 - 4 } & MFA & $0 \underline{\underline{a}}$ &. \\
\hline \multirow{2}{*}{ Ankle/feet } & ManTRA & -1.764 & $<0.001$ \\
\cline { 2 - 4 } & MFA & $0 \underline{\underline{a}}$ &. \\
\hline
\end{tabular}

Table 4: Disagreement between the three methods using Log-linear test.

The workers' information about musculoskeletal discomforts/pains gathered using the Cornell Musculoskeletal Discomfort Questionnaire. As shown in Figure 2, the percentage of the workers' musculoskeletal discomforts/pains, for each body part, are stratified in four categories as mild, moderate, fairly severe and severe discomfort. As can be seen, the prevalence of the back pain was greatest. It did not exceed $40 \%$ of the total workers' population, though. The least complaints, on the other hand, were made relative to the arms/elbows.

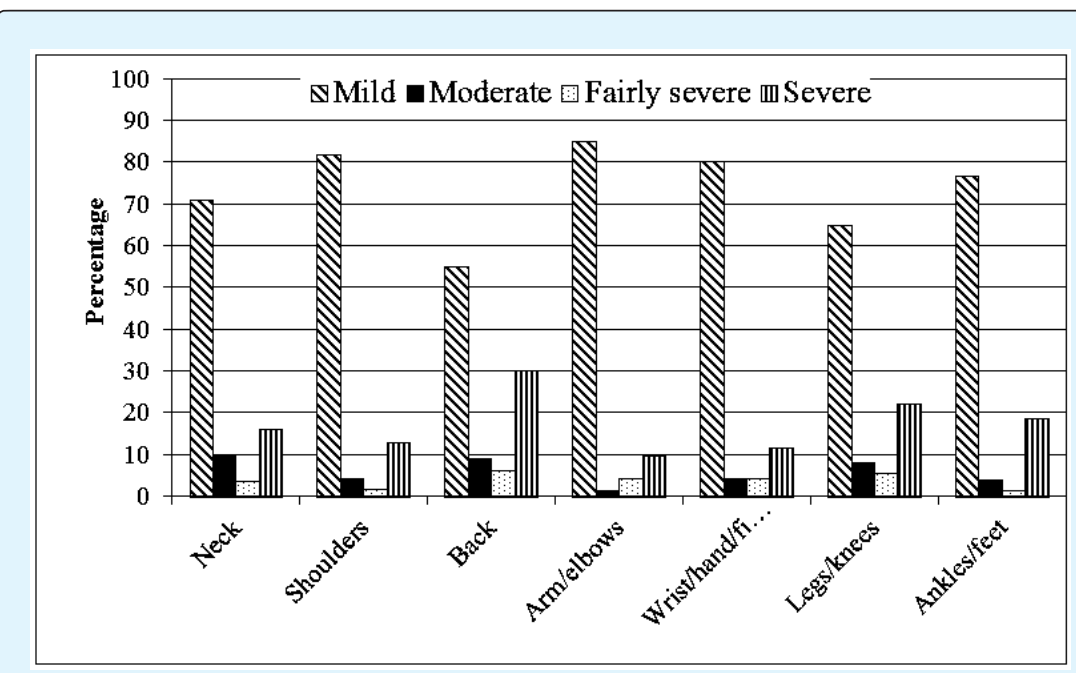

Figure 2: The workers' report of musculoskeletal discomforts. 


\section{Ergonomics International Journal}

The Pearson Chi-Square and Gamma tests at a significance level of 0.05 were used to find the significant correlation between the results of each method and the musculoskeletal discomfort questionnaire's responses with respect to each corresponding body region. Gamma test is applicable when ordinal by ordinal data is in hand
[27]. That is why; it was not applied for the analysis of the results of ManTRA because of its two-point scoring modality (ManTRA just determines whether or not a task needs to be modified) against four-scale pain score of Cornell questionnaire. It was examined just by Pearson Chi-Square test.

\begin{tabular}{|c|c|c|c|c|c|c|c|}
\hline & Neck & Shoulders & Back & Arms & Wrist/hands & Legs/knees & Ankles/feet \\
\hline QEC & 0.065 & 0.066 & $<0.001$ & 0.018 & 0.001 & - & - \\
\hline ManTRA & 0.016 & $<0.001$ & 0.051 & 0.016 & 0.020 & 0.214 & 0.49 \\
\hline MFA & 0.047 & $<0.001$ & $<0.001$ & $<0.001$ & $<0.001$ & 0.002 & $<0.001$ \\
\hline
\end{tabular}

Table 5: The P-Value of the Pearson Chi-Square test at a significance level of 0.05 .

\begin{tabular}{|c|c|c|c|c|c|c|c|}
\hline & Neck & Shoulders & Back & Arms & Wrists/hands & Legs/knees & Ankles/feet \\
\hline QEC & 0.012 & $<0.001$ & 0.016 & 0.091 & $<0.001$ & - & - \\
\hline MFA & 0.033 & $<0.001$ & $<0.001$ & $<0.001$ & $<0.001$ & 0.020 & $<0.001$ \\
\hline
\end{tabular}

Table 6: The P-Value of the ordinal by ordinal gamma at a significance level of 0.05 .

\section{Discussion}

In case of a particular work type assessment; there may be some similar-purpose methods which make the practitioners confuse to choose the most appropriate one. QEC, ManTRA and MFA are of those have to do with whole-body manual tasks assessment which their distinctions have not been studied yet. In this study, the results of assessing the risks of 350 manual labors using the three methods were compared with both each other and the workers' musculoskeletal discomforts with respect to each corresponding body regions.

As a result, MFA stratified the tasks in more moderate way than did either of the other two and had statistically significant correlation with the worker's musculoskeletal discomforts in all body regions. That is to say, most of the workstations whose workers experienced, at least, severe discomforts one to two times during last week were identified as high or very high risk by MFA method. Also as an outcome of a relevant study, in which it was proved to be consistent with a virtual, biomechanical analysis tool, a practically beneficial technique, MFA is a useful method to find biomechanical risks of musculoskeletal disorders [28].

ManTRA is found to be the most lenient tool of the three. In other words, it appears that its sensitivity is less than that of the other tools in identifying at risk tasks and tended to show false-negative results especially in back, but its leniency drops in arms/hands. The difference is so much that this method generally accorded neither with QEC nor with MFA. QEC, on the contrary, seemed to be stricter. In this study, although the complaints of discomforts in arms were the lowest, QEC could not make a significant correlation because of its stringency. Indeed, the outcomes of the relevant studies in this regard are consistent with our findings that there is clear distinction between the results of QEC and ManTRA. ALICI, et al. reported that the evaluated tasks are considered to be high riskier by QEC method than ManTRA [29]. Bell and Steele also found that ManTRA classified the vacuum cleaning tasks as at risk only in arms and hands and as safe in terms of back and neck, whereas QEC resulted that there were no safe task [30].

To be more elaborate, in this research, no correlation (neither disagreement, nor agreement) was statistically found between QEC and MFA in terms of back, Arms and Wrists/hands assessments. Even though, the results of ManTRA and MFA were relatively closer in neck, as were those of QEC and MFA in shoulders. The methods all are inversely correlated with one another in these body regions. it is noteworthy that although the results of two different methods may be close to each other (i.e. risk of arms by QEC and that by MFA), it should not be taken to mean that there must be a positive correlation between them, or vice versa. This is because what is graphed as a risk percentage of a given body part, is considered generally. While the statistical tests used case-by-case analysis to estimate the correlation of the results. Also a dramatic difference is seen in lower-limbs assessment between MFA and ManTRA which may have been due to their discrepancies in the way they consider the factors such as duration and repetition. To be precise, ManTRA evaluated $9 \%$ of the tasks as high risk in terms of lower limbs versus $55-57 \%$ resulted by MFA. This is while 23$35 \%$ of the workers felt pain in the lower limbs just during the last seven work days. It is important to note 


\section{Ergonomics International Journal}

that, in real practice, if a task assessment method in identifying musculoskeletal risks goes much further than what the workers report their musculoskeletal pains, could be acceptable. It is because despite the presence of the risks, work-related musculoskeletal traumas take time to show up. As a converse case, it would be undesirable.

Broadly speaking, it could be inferred that the risk factors and their weights each tool considers in its assessment impress the assessment outcome. The more similar risk factors a couple of methods take into account, the closer their final results are. This is consistent with what Jones and Kumar concluded from their study [4]. Each of the three tools weighs the considered risk factors differently. Of particular difference is the way they take the "duration" and "frequency/repetition" into account. QEC uses specific scales with respect to each body region to calculate the repetition of risk factors and a general scale to calculate the total exposure time for all. ManTRA applies an equivalent scale for all body regions to consider the repetition, duration and total exposure time with wider ranges of timeframes than the others two, which made it more permissive. MFA, however, calculates the "duration" and "frequency" of a particular effort into consideration with a narrow timeframe and does not take the total exposure time in a typical workday into consideration. MFA also takes posture, force and load lumped together as one, whereas the others evaluate them separately. Such discrepancies made these tools different from each other. Another possible reason for the difference, especially between the results of ManTRA and those of the other methods, could be that both QEC and MFA have parts in which the worker's participation is required for assessment, whereas ManTRA does not. That means, ManTRA is non-invasive, as well as easiest to use than the other two.

In this study, it was hard to find employees working at one task for a long time, because the workers had been rotated through the different tasks a couple of times. Therefore, just minimum one-year-work experienced workers were taken. Another limitation was to persuade the workers to be honest about expressing their musculoskeletal pains. It is yielded after a couple of attempts, though. As a future study, it is suggested to determine the consistency and sensitivity of the tools with respect to each work type.

\section{Conclusion}

This study has found that, of the three ergonomic assessment methods, MFA has more moderate results and is more predictive of workers' musculoskeletal pains. This tool is sensitive enough to evaluate the workers' muscle fatigue and could be suitable for repetitive tasks.

\section{Acknowledgement}

This study was supported by Mazandaran University of Medical Sciences, Iran (grant number IR.MAZUMS.REC.1396.10388). The authors would like to acknowledge the collaboration of all industries and those who assisted us in the process of data collection.

\section{References}

1. Jones T, Kumar S (2006) Assessment of physical demands and comparison of multiple exposure definitions in a repetitive high risk sawmill occupation: Saw-filer. Int J Ind Ergonom 36(9): 819827.

2. Ahmadi M, Zakerian SA, Salmanzadeh H, Mortezapour A (2017) Identification of the Ergonomic Interventions Goals from the Viewpoint of Ergonomics Experts of Iran using Fuzzy Delphi Method. Int J Occup Hyg 8(3): 151-157.

3. Landau KL, NadeauS, LeFloch T (2018) Development of the Ergonomic Activity Sampling (EAS) Method to Analyse Video-Documented Work Processes with Activity Sampling. Ergonomics Int J 1(2): 101.

4. Jones T, Kumar S (2010) Comparison of ergonomic risk assessment output in four sawmill jobs. Int J Occup Saf Ergon 16(1): 105-111.

5. Zarea M, Biau S, Brunet R, Roquelaure Y (2015) Are there differences between various ergonomic risk evaluation methods? observational methods, questionnaire and direct measurement. Proceedings 19th Triennial Congress of the IEA, Australia.

6. Genaidy AM, Al-Shedi AA, Karwowski W (1994) Postural stress analysis in industry. Appl Ergon 25(2): 77-87.

7. KeikhahMoghadam AA (2013) Ergonomics assessment methods; selection and application guidephysical assessment methods. Fanavaran, Tehran, Iran.

8. Villarroya A, Arezes P, Díaz-Freijo S, Fraga F (2016) Comparison between five risk assessment methods of patient handling. Int J Ind Ergonom 52: 100-108. 


\section{Ergonomics International Journal}

9. Hamrick C (2006) 3/Overview of ergonomic assessment. In: Karwowski W, Marras WS, (Eds.), The Occupational Ergonomics Handbook. Fundamentals and Assessment Tools for Occupational Ergonomics. $2^{\text {nd }}($ Edn.), Crc Press, Taylor \& Francis Group, Boca Raton, USA, pp: 1-2.

10. Jones T, Kumar S (2007) Comparison of ergonomic risk assessments in a repetitive high-risk sawmill occupation: Saw-filer. Int J Ind Ergonom 37(9): 744753.

11. Russell SJ, Winnemuller L, Camp JE, Johnson PW (2007) Comparing the results of five lifting analysis tools. Appl Ergon 38(1): 91-97.

12. Burgess-Limerick R (2003) Issues associated with force and weight limits and associated threshold limit values in the physical handling work environment. Issues Paper, Uni Quest Pty Ltd, pp: 21-22.

13. David G, Woods V, Li G, Buckle P (2008) The development of the Quick Exposure Check (QEC) for assessing exposure to risk factors for work-related musculoskeletal disorders. Appl Ergon 39(1): 57-69.

14. Comper ML, Costa LO, Padula RS (2012) Quick Exposure Check (QEC): a crosscultural adaptation into Brazilian-Portuguese. Work 41(S1): 2056-2059.

15. Bulduk EÖ, Bulduk S, Süren T, Ovalı F (2014) Assessing exposure to risk factors for work-related musculoskeletal disorders using Quick Exposure Check (QEC) in taxi drivers. Int J Ind Ergonom 44(6): 817-820.

16. Farhadi R, Omidi L, Balabandi S, Barzegar S, Abbasi $\mathrm{AM}$, et al. (2014) Investigation of musculoskeletal dsorders and its relevant factors using quick exposure check (QEC) method among seymareh hydropower plant workers. J Research Health 4(2): 714-720.

17. Li G, Buckle P (1998) A practical method for the assessment of work-related musculoskeletal risksQuick Exposure Check (QEC). Proceedings of the human factors and ergonomics society annual meeting, USA.

18. Li G, Buckle P (2004) 6/ Quick Exposure Checklist (QEC) for the Assessment of Workplace Risks for Work-Related Musculoskeletal Disorders (WMSDs). In: Stanton NA, Hedge A, Brookhuis K, Salas E, Hendrick HW, (Eds.), Handbook of human factors and ergonomics methods. Crc Press, Taylor \& Francis Group, Boca Raton, USA, pp: 1-7.

19. Abdollahpour N, Helali F, Ziaei M, Hamzeian M, Keikhamoghadam AA, et al. (2016) Assessment of risk factors and the prevalence of musculoskeletal disorders by QEC and Body Map operational units of the gas refinery in Iran and its Meta-Reflection. J Health 7(1): 35-50.

20. Burgess-Limerick R, Straker L, Pollock C, Egeskov R (2004) Manual Tasks Risk Assessment Tool (ManTRA) V 2.0. Human Factors and Ergonomics Society of Australia workshop at the Human Factors and Ergonomics Society of Australia annual conference, Australia.

21. Moussavi-Najarkola SA, Mirzaei R (2012) ManTRA for the Assessment of Musculoskeletal Risk Factors Associated With Manual Tasks in an Electric Factory. Health Scope 1(3): 132-139.

22. Motamedzade M, Saedpanah K, Eskandarian T, Salimi $\mathrm{K}$ (2016) Risk assessment of musculoskeletal disorders by Muscle Fatigue Assessment method and implementation of an ergonomic intervention in Assembly industry. J Occup Hyg Eng 3(1): 33-40.

23. Kalte HO (2016) Evaluation of Work Fatigue in Loading Workers Using Muscle Fatigue Assessment Method (MFA): A Case Study in a Brick Factory. J health Res Com 2(2): 29-36.

24. Hedge A (1999) Cornell Musculoskeletal Discomfort Questionnaires. Cornell University Ergonomics.

25. Afifehzadeh-Kashani H, Choobineh A, Bakand S, Gohari MR, Abbastabar H, et al. (2011) Validity and reliability of farsi version of Cornell Musculoskeletal Discomfort Questionnaire (CMDQ). Iran Occup Health 7(4): 69-75.

26. Johnson S, McLeod K, Engel P, Tulloch L (2016) Musculoskeletal Disorders and Health Profile of Continuing Care Aides: An Urban-Rural Comparison. Home Health Care Manag Pract 28(3): 135-141.

27. Agresti A (2013) Categorical data analysis. $2^{\text {nd }}$ (Edn.), John Wiley \& Sons, Hoboken, New Jersey, USA.

28. Hernandez-Arellano JL, Serratos-Perez JN, de la Torre A, Maldonado-Macias AA, Garcia-Alcaraz JL (2015) Design proposal of an adjustable workstation for very short and very tall people. Procedia Manuf 3: 56995706. 


\section{Ergonomics International Journal}

29. Alici H, Ulusu Ha, Gündüz T (2017) Mobilya Sektöründe Pnömatik Zımbalama ve Vidalama İşlerinin Ergonomik Risk Değerlendirmesi. Çukurova Üniversitesi Mühendislik-Mimarlık Fakültesi Dergisi. 32(4): 211-226.
30. Bell AF, Steele JR (2012) Risk of musculoskeletal injury among cleaners during vacuuming. Ergonomics 55(2): 237-247. 